\title{
Thermodynamic Analysis of a Variable Viscosity Reactive Hydromagnetic Couette Flow within Parallel Plates
}

\author{
Anthony R. Hassan", Olufemi W. Lawal and Funmilayo F. Amurawaye \\ Department of Mathematics, Tai Solarin University of Education, Ijagun, Ogun State, Nigeria. \\ "Corresponding author, E-mail: anthonyhassan72@yahoo.co.uk \\ Co-authors'e-mails: waheedlawal207@yahoo.com; amurawayeff@tasued.edu.ng \\ Received 29 Sep 2020, Revised 9 Mar 2021, Accepted 21 Apr 2021, Published May 2021
}

DOI: https://dx.doi.org/10.4314/tjs.v47i2.3

\begin{abstract}
This investigation is to consider the impact of a temperature-dependent variable viscosity of a reactive hydromagnetic Couette fluid flowing within parallel plates. The variable property of the fluid viscosity is thought to be an exponential relation of temperature under the impact of magnetic strength. The differential equations controlling the smooth movement of fluid and energy transfer are modeled and solved by using the series solution of modified Adomian decomposition technique (mADM). The outcomes are shown in tables and graphs for different estimations of thermophysical properties present in the flow regime together with the rate of entropy generation and irreversibility distribution outcome.
\end{abstract}

Keywords: Reactive fluids, Couette Flow, variable viscosity, hydromagnetic and modified Adomian decomposition method (mADM).

\section{Introduction}

The uses of non-Newtonian fluids in current innovations in industries have been tremendous and attractive to researchers because of their essentialness in the current day industrial and engineering procedures. Many problems concerning the fluid flow with thermophysical properties which sometimes are assumed to be constant, some of these properties may alter fluid temperature, most importantly, the fluid viscosity. As discussed in Hassan (2019), the prediction of heat transfer rate and flow speed completely, it is critical to consider the variation of viscosity with respect to temperature.

However, the study of Couette flow is remarkably necessary because of its industrial applications in numerous flows occurring in many procedures that have been modeled with diverse heat transfer circumstances. Therefore, such complex fluid flow has been comprehensively investigated by many researchers for different limit conditions and other effects, for instance, the investigation of the entropy generation in an unsteady MHD Couette flow of a viscous incompressible electrically conducting fluid with respect to magnetic field strength in a circular system conducted in Das et al. (2016a). In addition to that, Das et al. (2016b) further examined the compound impacts of spinning and the magnetic field on the transient MHD Couette flow of a reactive fluid in a rotating system. To buttress the significant study on Couette flow, Makinde et al. (2015) employed the first two laws of thermodynamics to examine the flow and thermal dissipation in MHD variable viscosity flow of a Couette flow in a spinning system. Other investigations on Couette flow and other physical properties are extensively presented in Makinde (2014), Theuri and Makinde (2014), and Vyas and Ranjan (2015). 432 
Notably, the investigation of Hayat et al. (2016) on temperature dependent thermal conductivity in a stretched surface with variable thickness in stagnation flow highlighted the impact of chemical reaction between fluid and foreign mass.

Moreover, to prevent a danger or risk in a system that controls fluid behavior, it is imperative to take note of fluid flow and heat transfer conditions, particularly the fluid viscosity. It is well known that viscous heating is generated due to surface interaction between the fluid and the walls together with high shear rates that lead to high temperature; in actual fact, viscosity is the most delicate fluid property that causes change in temperature as supported and complimented in Makinde and Maserumule (2008). Hence, it is important to note that the diverse means of fluid viscosity due to change in temperature may remarkably affect the flow properties together with efficiency of lubrication in industrial tools. Many authors have investigated various models of variable viscosity of fluid flow characteristics, for instance, Eegunjobi et al. (2015) examined the compound effects of unsteady and temperature-dependent viscosity on the rate of entropy distribution in a generalized Couette flow. Additionally, Makinde (2008a) considered the consequences of variable viscosity fluid through parallel channels with convective cooling the walls with the temperature-dependent viscosity as an exponential function of temperature. In addition to that, Hassan (2019) examined the impact of heat source without magnetic strength influence on a variable viscosity reactive Couette fluid flow. Further studies involving properties of variable viscosity could be found (Salem 2007, Mukhopadhyay 2009, Kobo and Makinde 2010, Hassan and Gbadeyan 2013, Makinde et al. 2016a, Makinde et al. 2016b, Salawu and Oke 2018, Wang et al. 2020).

From application point of view, the present study is to determine the impact of variable viscosity on a reactive hydromagnetic Couette fluid between parallel moving upper and static lower plate with an exponential temperaturedependent viscosity. The impact of electromagnetic influence on any fluid flow is highly noticeable on a flow of viscous materials as extensively discussed in Salem (2007), Hassan et al. (2017), and Muhammad et al. (2020). The model problem is obtained and solved analytically using a series solution of modified Adomian decomposition method (mADM). The choice of this technique is based on the rapid convergence with less iteration and has been shown in literature (Adomian 1994, Wazwaz 1999, Wazwaz and El-Sayed 2001) to be effective and accurate. The expressions from the outcomes of velocity and heat transfer are employed to determine the rate of entropy distribution, irreversibility distribution ration and Bejan number. Pertinent outcomes are shown in tables and graphs to demonstrate the remarkable impact of magnetic field strength and other thermophysical properties in the flow stream.

\section{Problem Formulations}

We consider a steady flow of an incompressible, reactive, viscous and hydromagnetic fluid with moving upper plate and stationary lower plate as shown in the configuration in Figure 1. The fluid viscosity is assumed to be an exponential function of temperature between two parallel plates of width, $H$ and length, $L$. The upper plate is driven with uniform speed, $U$, while the lower plate is kept unmoved.

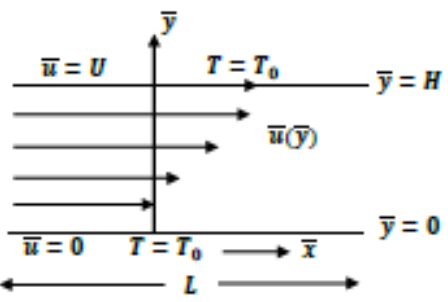

Figure 1: Geometry of the problem

The variable property of the fluid viscosity is taken to be an exponential function of temperature as used in Makinde (2008b), Makinde and Maserumule (2008), Gbadeyan 
and Hassan (2012), Eegunjobi et al. (2015), Makinde et al. (2015) and Hassan (2019) as:

$\bar{\mu}=\mu_{0} e^{-\frac{E}{R \bar{T}}}$

where $\mu_{0}, E, R$ and $T$, respectively stand for the fluid reference dynamic viscosity at a very large temperature (i.e. as $T \rightarrow \infty$ ), the activation energy, the universal gas constant and the absolute temperature.

The equations controlling the fluid flow in dimensional form without the reactant consumption are given as described in Makinde and Maserumule (2008), Kobo and Makinde (2010), and Hassan (2019) as: $\frac{d}{d \bar{y}}\left(\bar{u} \frac{d \bar{u}}{d \bar{y}}\right)-\sigma \frac{\beta_{0}^{2}}{\rho} \bar{u}=0$

$k \frac{d^{2} \bar{T}}{d \overline{y^{2}}}+\bar{\mu}\left(\frac{d \bar{u}}{d \bar{y}}\right)^{2}+Q C_{0} A e^{-\frac{E}{R \bar{T}}}+\sigma \frac{\beta_{0}^{2}}{\rho} \bar{u}^{2}=0$

such that the initial boundary situations are given as:

$\bar{u}(0)=\bar{T}(0)=0, \quad \bar{u}(1)=1 \quad$ and

$\bar{T}(1)=0$

We thereby introduce the dimensionless variables and parameters as follows:

$$
\begin{aligned}
& y=\frac{\bar{y}}{L}, \quad x=\frac{\bar{x}}{L}, u=\frac{\bar{u}}{u}, \quad \mu=\frac{\bar{\mu}}{\mu_{0}} e^{-\frac{E}{R T}}, \quad \theta=\frac{E\left(\bar{T}-T_{0}\right)}{R T_{0}^{2}}, \quad \in=\frac{R T_{0}}{E}, \Omega=\frac{E}{R T_{0}}, \\
& B r=\frac{\mu_{0} E U}{k R T_{0}^{2}}, \quad \gamma=\frac{\mu_{0} E U}{k R T_{0}^{2}} e^{-\frac{E}{R T_{0}}}, \quad H^{2}=\frac{\sigma B_{0}^{2} L^{2}}{\rho} \text { and } \lambda=\frac{Q C_{0} E A L^{2}}{K R T_{0}^{2}} e^{-\frac{E}{R T_{0}}}
\end{aligned}
$$

where $(x, y)$ are distances measured in stream wise and normal directions, respectively, $\mu$ is the dynamic viscosity, $k$ represents the thermal conductivity, $u$ is the axial velocity, $U$ is the velocity scale, $\in$ is the activation energy parameter, $\lambda$ is the Frank-Kamenettski parameter. However, the flow at the upper plate is driven by a constant velocity without the pressure gradient.

$$
\begin{aligned}
& \frac{d}{d y}\left(\mu \frac{d u}{d y}\right)-H^{2} u=0 \\
& \frac{d^{2} \theta}{d y^{2}}+\lambda\left(e^{\frac{\theta}{1+\epsilon \theta}}+\gamma\left(\mu\left(\frac{d u}{d y}\right)^{2}+H^{2} u^{2}\right)\right)=0
\end{aligned}
$$

with the appropriate boundary conditions as follows:

$$
\begin{aligned}
& u(0)=\theta(0)=0 \quad \text { and } \quad u(1)=1 \quad \text { and } \\
& \theta(1)=0 \quad \text { (8) }
\end{aligned}
$$

such that, the dynamic viscosity is given as $\mu=e^{-\frac{\theta}{1+\in \theta}}$

\section{Solution Method}

The governing Equations (6) and (7) are non-linear differential equations (ODE) with no exact analytical solution. Therefore, for accuracy, effectiveness, better stability and simplicity, we employed the use of series solution of modified Adomian decomposition method by assuming that the outcomes appear in the following forms:

$$
u(y)=\sum_{n=0}^{\infty} u_{n}(y) \text { and } \theta(y)=\sum_{n=0}^{\infty} \theta_{n}(y)
$$

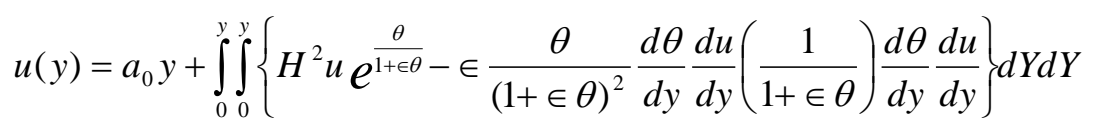


$\theta(y)=b_{0} y-\lambda \int_{0}^{y} \int_{0}^{y}\left\{e^{\frac{\theta}{1+\epsilon \theta}}+\gamma\left[e^{-\frac{\theta}{1+\epsilon \theta}}\left(\frac{d u}{d y}\right)^{2}+H^{2} u^{2}\right]\right\} d Y d Y$

where $a_{0}$ and $b_{0}$ are constants of integration

solutions introduced in Equation (9), the to be resolved by putting to use the limit Equations (10) and (11) reduce to the conditions in Equation (8). With the series

$$
\begin{aligned}
& u(y)=a_{0} y+\int_{00}^{y y}\left\{H^{2} \sum_{n=0}^{\infty} A_{n}-\epsilon \sum_{n=0}^{\infty} B_{n}-\sum_{n=0}^{\infty} C_{n}\right\} d Y d Y \\
& \theta(y)=b_{0} y-\lambda \iint_{00}^{y y}\left\{\sum_{n=0}^{\infty} D_{n}+\gamma\left[\sum_{n=0}^{\infty} E_{n}+H^{2} \sum_{n=0}^{\infty} F_{n}\right]\right\} d Y d Y
\end{aligned}
$$

where the non-linear terms present are termed respectively as follows:

$$
\begin{aligned}
& \sum_{n=0}^{\infty} A_{n}(y)=\sum_{n=0}^{\infty} \mathrm{u}_{\mathrm{n}} e \frac{\sum_{n=0}^{\infty} \theta_{n}}{1+\in \sum_{n=0}^{\infty} \theta_{n}}, \sum_{n=0}^{\infty} B_{n}(y)=\frac{\sum_{n=0}^{\infty} \theta_{n}}{\left(1+\epsilon \sum_{n=0}^{\infty} \theta_{n}\right)^{2}} \frac{d \sum_{n=0}^{\infty} \theta_{n}}{d y} \frac{d \sum_{n=0}^{\infty} u_{n}}{d y}, \\
& \sum_{n=0}^{\infty} C_{n}(y)=\left(\frac{1}{1+\in \sum_{n=0}^{\infty} \theta_{n}}\right) \frac{d \sum_{n=0}^{\infty} \theta_{n}}{d y} \frac{d \sum_{n=0}^{\infty} u_{n}}{d y}, \sum_{n=0}^{\infty} D_{n}(y)=e \frac{\sum_{n=0}^{\infty} \theta_{n}}{1+\in \sum_{n=0}^{\infty} \theta_{n}}, \\
& \sum_{n=0}^{\infty} E_{n}(y)=e-\frac{\sum_{n=0}^{\infty} \theta_{n}}{1+\in \sum_{n=0}^{\infty} \theta_{n}}\left(\frac{d \sum_{n=0}^{\infty} u_{n}}{d y}\right) \quad \text { and } \sum_{n=0}^{\infty} F_{n}(y)=\sum_{n=0}^{\infty} \mathrm{u}_{\mathrm{n}}^{2} .
\end{aligned}
$$

such that the Adomian polynomials, $A_{0}, A_{1}$, expanding each expression in Equation (14) $A_{2}, \ldots \ldots$ to $F_{0}, F_{1}, F_{2}, \ldots \ldots$ are obtained by such that few are stated thus:

$$
\begin{aligned}
& A_{0}=u_{0}(y) e^{\frac{\theta_{0}(y)}{\epsilon \theta_{0}(y)+1}}, \quad A_{1}=\frac{e^{\frac{\theta_{0}(y)}{\epsilon \theta_{0}(y)+1}}\left(u_{0}(y) \theta_{1}(y)+u_{1}(y)\left(\in \theta_{0}(y)+1\right)\right)^{2}}{\left(\in \theta_{0}(y)+1\right)^{2}}, \ldots, \\
& B_{0}=\frac{\theta(y) u_{0}^{\prime}(y) \theta_{0}^{\prime}(y)}{\left(\in \theta_{0}(y)+1\right)^{2}}, \\
& B_{1}=\frac{\left(u_{0}^{\prime}(y) \theta_{1}(y) \theta_{0}^{\prime}(y)\left(1-\in \theta_{0}(y)\right)+\theta_{0}(y)\left(\in \theta_{0}(y)+1\right)\right)\left(u_{1}^{\prime}(y) \theta_{0}^{\prime}(y)+u_{0}^{\prime}(y) \theta_{1}^{\prime}(y)\right.}{\left(\in \theta_{0}(y)+1\right)^{3}}, \ldots \\
& C_{0}=\frac{u_{0}^{\prime}(y) \theta_{0}^{\prime}(y)}{\in \theta_{0}(y)+1}, \quad C_{1}=\frac{\left(\in \theta_{0}(y)+1\right)\left(u_{1}^{\prime}(y) \theta_{0}^{\prime}(y)+u_{0}^{\prime}(y) \theta_{1}^{\prime}(y)-\in \theta_{0}^{\prime}(y) u_{0}^{\prime}(y) \theta_{1}(y)\right)}{\left(\in \theta_{0}(y)+1\right)^{2}}, \ldots \ldots . . \\
& D_{0}=\frac{\theta_{0}(y)}{e^{\epsilon \theta_{0}(y)}+1}, \quad D_{1}=\frac{\theta_{1}(y) e^{\frac{\theta_{0}(y)}{\epsilon \theta_{0}(y)+1}}}{\left(\in \theta_{0}(y)+1\right)^{2}}, \ldots, E_{0}=u_{0}^{\prime 2}(y) e^{-\frac{\theta_{0}(y)}{\epsilon \theta_{0}(y)+1}},
\end{aligned}
$$




$$
\begin{aligned}
& E_{1}=\frac{u_{0}^{\prime}(y) e^{-\frac{\theta_{0}(y)}{\epsilon \theta_{0}(y)+1}\left(2 u_{1}^{\prime}(y)\left(\in \theta_{0}(y)+1\right)^{2}-\theta_{1}(y) u_{0}(y)\right)}}{\left(\in \theta_{0}(y)+1\right)^{2}}, \ldots, F_{0}=u_{0}(y), \\
& F_{1}=2 u_{0}(y) u_{1}(y), \ldots
\end{aligned}
$$

With the Adomian polynomials obtained in Equation (15), we take the zeroth components of the couple Equations (12) and (13) as

$$
\begin{aligned}
& u_{0}=a_{0}(y), \quad \theta_{0}=0 \\
& u_{1}(y)=\int_{00}^{y} \int_{0}^{y}\left\{H^{2} A_{0}(y)-\in B_{0}(y)-C_{0}\right\} d Y d Y \\
& \theta_{1}(y)=b_{0} y-\lambda \int_{0}^{y} \int_{0}^{y}\left\{D_{0}(y)+\gamma\left[E_{0}(y)+H^{2} F_{0}\right]\right\} d Y d Y \quad \forall n \geq 1 \\
& \left.u_{n+1}(y)=\int_{00}^{y} \int_{0}^{y}\left\{H^{2} A_{n}(y)-\in B_{n}(y)-C_{n}(y)\right\} d Y d Y \quad \forall n E^{2} F_{n}(y)\right] d Y d Y \\
& \theta_{n+1}(y)=-\lambda \int_{0}^{y} \int_{0}^{y}\left\{D_{n}(y)+\gamma\left[E_{n}(y)+H^{2}\right.\right.
\end{aligned}
$$$$
\text { described in Wazwaz and El-Sayed (2001), and }
$$
Hassan et al. (2017) for the solutions as follows:
Equations (16) to (20) are hereby done repeatedly to acquire the approximate results for momentum and energy distributions as:

$$
u(y)=\sum_{n=0}^{k} u_{n}(y) \text { and } \theta(y)=\sum_{n=0}^{k} \theta_{n}(y)
$$

\section{Entropy Generation}

Flow and heat transfer processes within parallel channels interact with each other and thereby causing the exchange of thermal energy within the flow stream, thus resulting in the rate of entropy production. Following Wood (1975), Bejan (1996), Makinde (2008), and Hassan and Gbadeyan (2015), the equation for finding the rate of entropy generation together with the influence of magnetic strength is presented as:

$$
S^{m}=\frac{k}{T_{0}^{2}}\left(\frac{d \bar{T}}{d \bar{y}}\right)^{2}+\frac{\bar{\mu}}{T_{0}}\left[\left(\frac{\partial \bar{u}}{\partial \bar{y}}\right)^{2}+\sigma \frac{\beta_{0}}{\rho} \bar{u}^{2}\right]
$$

The terms in Equation (22) show the irreversibility caused by heat transfer and the local entropy production caused by viscous dissipation and magnetic strength, respectively. Using the existing parameters and variables in
Equation (5), the rate of entropy generation is given as:

$$
N_{s}=\frac{S^{m} L^{2} T_{0}^{2}}{T_{0}^{2}}=\left(\frac{d \theta}{d y}\right)^{2}+\frac{B r}{\Omega}\left[\mu\left(\frac{\partial u}{\partial y}\right)^{2}+H^{2} u^{2}\right]
$$

For convenience, Equation (23) is divided into two portions, $N_{1}$ and $N_{2}$ to respectively represent the irreversibility due to heat transfer and effects of viscous dissipation together with magnetic strength. With that we have

$$
\begin{aligned}
& N_{1}=\left(\frac{d \theta}{d y}\right)^{2} \\
& N_{2}=\frac{B r}{\Omega}\left[\mu\left(\frac{\partial u}{\partial y}\right)^{2}+H^{2} u^{2}\right]
\end{aligned}
$$

and

However, we defined the irreversibility distribution ratio as:

$\phi=\frac{N_{1}}{N_{2}}$

such that heat transfer dominates when $0<\phi<1$ and fluid friction dominates when 
$\phi>1$. As an alternative to irreversibility parameter, the Bejan number $(B e)$ is defined as $B e=\frac{N_{1}}{N_{s}}=\frac{1}{1+\phi}$ where $0 \leq B e \leq 1$

\section{Results and Discussion}

In this section, we describe the outcomes from the coupled differential equations to determine the impact of magnetic strength on a temperature-dependent reactive variable viscosity Couette flow. Table 1 displays the rapid convergence of the series solution for the constants $a_{0}$ and $b_{0}$ in Equations (10) and (11) with little iteration to show the effectiveness of the series solutions of modified Adomian decomposition technique as already discussed in introduction. Table 2 displays the comparison of the numerical outcomes of temperature distribution obtained using perturbation method in investigation done in Kobo and Makinde (2010) and the present results obtained from modified decomposition method of Adomian (mADM). The results obtained from both methods demonstrate the agreement between the two methods with difference of order $10^{-3}$.

Table 1: Rapid convergence of the series solution for $a_{0}$ and $b_{0}$

\begin{tabular}{|c|c|c|}
\hline \multicolumn{3}{|c|}{$\gamma=\lambda=H=\in=0.1$} \\
\hline$n$ & $a_{0}$ & $b_{0}$ \\
\hline 0 & 1 & 0 \\
\hline 1 & 0.99834 & $0: 054992$ \\
\hline 2 & 1.00777 & $0: 055551$ \\
\hline 3 & 1.00760 & $0: 055426$ \\
\hline 4 & 1.00758 & $0: 055428$ \\
\hline 5 & 1.00758 & $0: 055428$ \\
\hline
\end{tabular}

Table 2: Comparison of numerical outcomes of the temperature profile

\begin{tabular}{|l|c|c|c|}
\hline \multicolumn{3}{|c|}{$\boldsymbol{H}=\mathbf{0}, \boldsymbol{\epsilon}=\mathbf{0 . 1}, \boldsymbol{\gamma}=\mathbf{0 . 1}, \boldsymbol{\lambda}=\mathbf{0 . 5}$} \\
\hline $\mathrm{Y}$ & $\mathrm{T}(\mathrm{y}) \mathrm{PM}($ Kobo and Makinde 2010) & $\theta(y) m A D M$ & Absolute error \\
\hline 0 & 0 & & 0 \\
\hline 0.1 & 0.02360044943 & 0.02475 & $1.14955 \times 10^{-3}$ \\
\hline 0.2 & 0.04208380022 & 0.04400 & $1.91620 \times 10^{-3}$ \\
\hline 0.3 & 0.05535532038 & 0.05775 & $2.39468 \times 10^{-3}$ \\
\hline 0.4 & 0.06334613033 & 0.06600 & $2.65387 \times 10^{-3}$ \\
\hline 0.5 & 0.06601440811 & 0.06875 & $2.73559 \times 10^{-3}$ \\
\hline 0.6 & 0.06334613023 & 0.06600 & $2.65387 \times 10^{-3}$ \\
\hline 0.7 & 0.05535532037 & 0.05775 & $2.39468 \times 10^{-3}$ \\
\hline 0.8 & 0.04208380020 & 0.04400 & $1.91620 \times 10^{-3}$ \\
\hline 0.9 & 0.02360044944 & 0.02475 & $1.14955 \times 10^{-3}$ \\
\hline 10 & 0 & 0 & 0 \\
\hline
\end{tabular}


Figure 2 displays the fluid motion with variation in magnetic strength parameter $(H)$. It is noticed that the flow speed remarkably reduces as $(H)$ rises. This is caused due to the presence of electromagnetic force across the channel and thus brings about the delay in the fluid flow.

The temperature profiles of the fluid system are with variations in the values of magnetic strength parameter $(H)$, Frank-Kamenettski parameter $(\lambda)$ and viscous heating parameter ( $\gamma$ ) are respectively displayed in Figures 3 to 5.

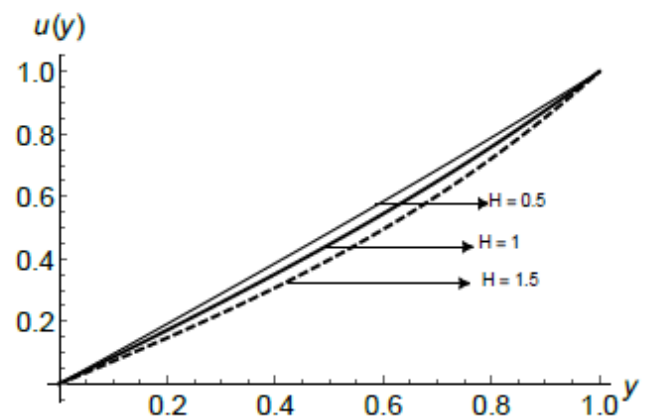

Figure 2: Effect of $H$ on $u(y)$

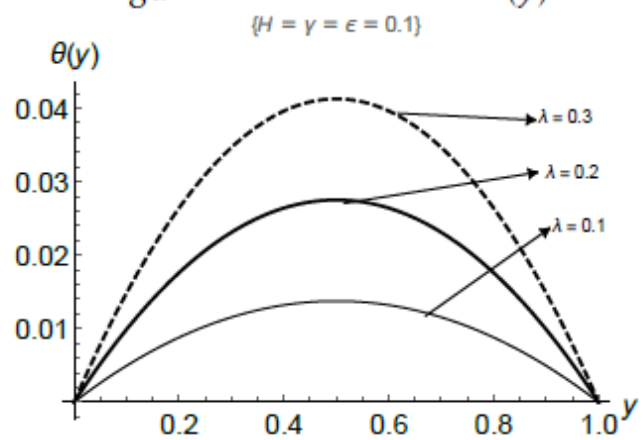

Figure 4: Effect of $\lambda$ on $\theta(y)$
The greatest temperature occurs at the centerline of the flow regime on a general note. It is noticed that the maximum temperature occurs with the least value of $(H)$ in Figure 3 and otherwise gives the greatest value of $(\lambda)$ in Figure 4 and in Figure 5. The increment in fluid temperature in Figure 4 and 5 is due to the overwhelming performance of variable viscosity properties that is an exponential relation of temperature.

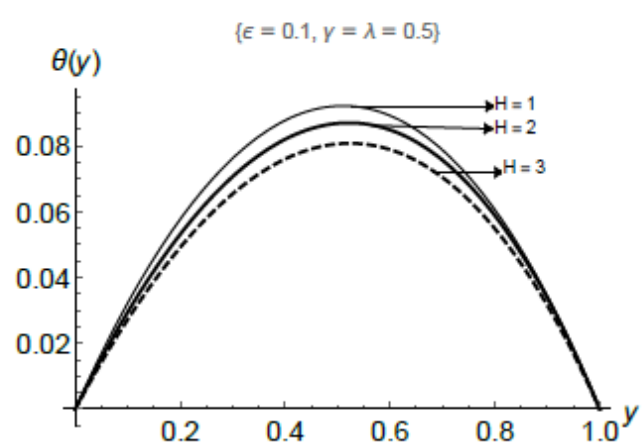

Figure 3: Effect of $H$ on $\theta(y)$

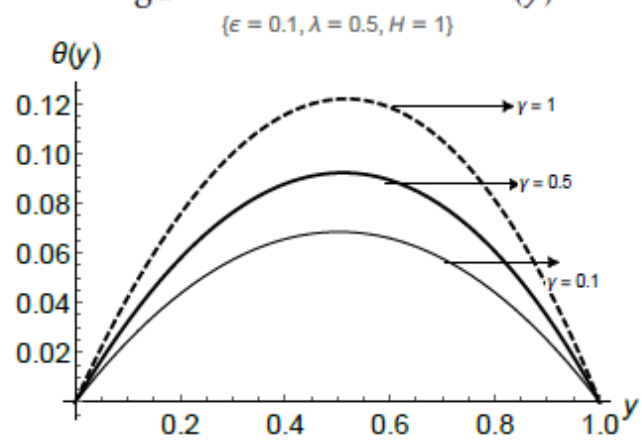

Figure 5: Effect of $\gamma$ on $\theta(y)$ 


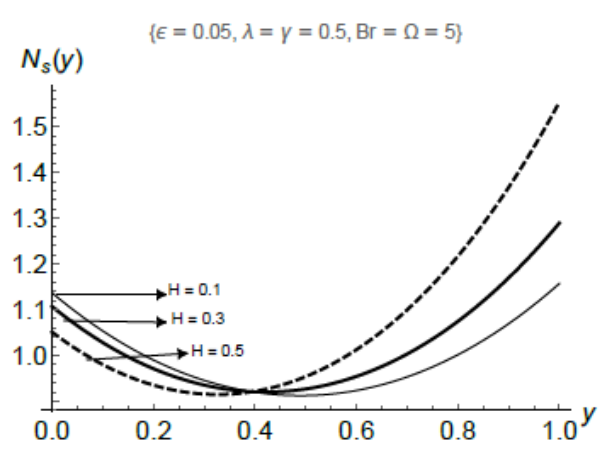

Figure 6: Effect of $H$ on $N_{s}(y)$

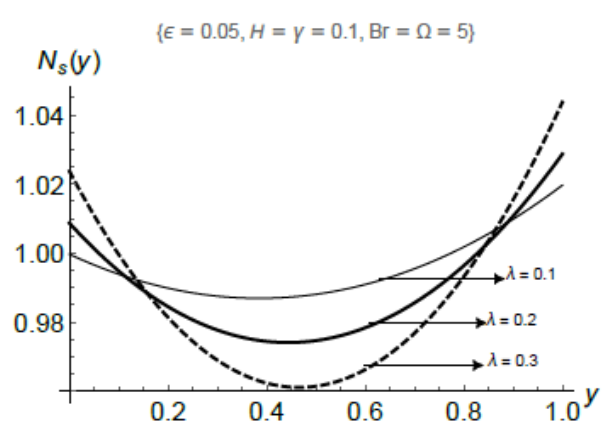

Figure 7: Effect of $\lambda$ on $N_{s}(y)$

\section{Conclusion}

The study conducted herein showed the significant effect of magnetic strength on a temperature dependent variable viscosity of reactive hydromagnetic coquette flow with fixed lower wall and moving upper wall that are both parallel. The coupled differential equations controlling the fluid flow are determined using the modified decomposition method of Adomian (mADM). The results obtained for momentum and energy distributions are used to determine the rate of entropy generation, the irreversibility distribution ratio and Bejan number. The results showed the meaningful impact of magnetic strength that reduces the fluid momentum together with fluid temperature. Also, the exponentially temperature-dependent variable viscosity contributed to the remarkable increase in fluid temperature with respect to viscous heating parameter and combustion parameter.

\section{References}

Adomian A 1994 Solving frontier problems of physics: the decomposition method. Kluwer academic Publishers, Boston.

Bejan A 1996 Entopy-generation minimization. New York CRC Press, New York.

Das S, Jana RN and Chamkha AJ 2016a Entropy generation due to unsteady hydromagnetic Couette flow and heat transfer with asymmetric convective 
cooling in a rotating system. J. Math. Model. 3(2): 111-128.

Das S, Jana RN and Makinde OD 2016b Transient hydromagnetic reactive Couette flow and heat transfer in a rotating frame of reference. Alexandria Eng. J. 55(1): 635644.

Eegunjobi AS, Makinde OD, Tshehla MS and Franks O 2015 Irreversibility analysis of unsteady Couette flow with variable viscosity. J. Hydrodynamics 27(2): 304 310.

Gbadeyan JA and Hassan AR 2012 Multiplicity of solutions for a reactive variable viscous Couette flow under Arrhenius kinetics. Math. Theor. Model. 2(9): 39-49.

Hassan AR 2019 Thermodynamics analysis of an internal heat generating fluid of a variable viscosity reactive Couette flow. $J$. King Saud University-Science 31: 506-510.

Hassan AR and Gbadeyan JA 2013 The effect of heat absorption on a variable viscosity reactive Couette flow under Arrhenius kinetics. Theor. Math. Appl. 3(1): 145-159.

Hassan AR and Gbadeyan JA 2015 Entropy generation analysis of a reactive hydromagnetic fluid flow through a channel. Univ. Politech. Buchar. Sci. Bull. Ser. A 77(2): 285-296.

Hassan AR, Maritz R and Gbadeyan JA 2017 A reactive hydromagnetic heat generating fluid flow with thermal radiation within porous channel with symmetrical convective cooling. Int. J. Thermal Sci. 122: 248-256.

Hayat T, Khan MI, Farooq M, Alsaedi A, Waqas $M$ and Yasmeen T 2016 Impact of Cattaneo-Christov heat flux model in flow of variable thermal conductivity fluid over a variable thicked surface. Int. J. Heat and Mass Transfer 99: 702-710.

Kobo NS and Makinde OD 2010 Second law analysis for a variable viscosity reactive Couette flow under Arrhenius kinetics. Math. Problems Eng. 2010: 1-15.

Makinde OD 2008a Entropy-generation analysis for variable-viscosity channel flow with non-uniform wall temperature. Appl. Energy 85: 384-393.

Makinde OD 2008b Irreversibility analysis of variable viscosity channel flow with convective cooling at the walls. Canadian J. Phys. 86(2): 383-389.

Makinde OD 2014 Thermal analysis of a reactive generalized Couette flow of power law fluids between concentric cylindrical pipes. Eur. Phys. J. Plus 129(12): 270.

Makinde OD, Eegunjobi AS, and Tshehla MS 2015 Thermodynamics analysis of variable viscosity hydromagnetic Couette flow in a rotating system with hall effects. Entropy 17(11): 7811-7826.

Makinde OD and Maserumule RL 2008 Thermal criticality and entropy analysis for a variable viscosity Couette flow. Physica Scripta 78: 015402.

Makinde OD, Khan WA, and Culham JR 2016a MHD variable viscosity reacting flow over a convectively heated plate in a porous medium with thermophoresis and radiative heat transfer. Int. J. Heat and Mass Transfer 93: 595-604.

Makinde OD, Mabood F, Khan WA and Tshehla MS 2016b MHD flow of a variable viscosity nanofluid over a radially stretching convective surface with radiative heat. J. Molecular Liquids 219: 624-630.

Muhammad R, Khan MI, Khan NB and Jameel M 2020 Magnetohydrodynamics (MHD) radiated nanomaterial viscous material flow by a curved surface with second order slip and entropy generation. Computer Methods and Programs in Biomedicine 189: 105294.

Mukhopadhyay S 2009 Effects of radiation and variable fluid viscosity on flow and heat transfer along a symmetric wedge. J. Appl. Fluid Mechanics 2(2): 29-34.

Salawu SO and Oke SI 2018 Inherent irreversibility of exothermic chemical reactive thirdgrade Poiseuille flow of a variable viscosity with convective cooling. J. Appl. Comput. Mechan. 4(3): 167-174.

Salem AM 2007 Variable viscosity and thermal conductivity effects on MHD flow and heat transfer in viscoelastic fluid over a 
stretching sheet. Phys. Lett. A 369(4): 315322.

Theuri D and Makinde OD 2014 Thermodynamic analysis of variable viscosity MHD unsteady generalized Couette flow with permeable walls. Appl. Comput. Math. 3(1): 1-8.

Vyas P and Ranjan A 2015 Entropy analysis for MHD generalized Couette flow in a composite duct. J. Industr. Math. 2015.

Wang J, Khan MI, Khan WA, Abbas SZ and Khan MI 2020 Transportation of heat generation/absorption and radiative heat flux in homogeneous-heterogeneous catalytic reactions of non-Newtonian fluid (Oldroyd-B model). Computer Methods and Programs in Biomedicine 189: 105310.

Wazwaz AM 1999 A reliable modification of Adomian decomposition method. Appl. Math. Comput. 102(1): 77-86.

Wazwaz AM and El-Sayed SM 2001 A new modification of the Adomian decomposition method for linear and nonlinear operators. Appl. Maths Comput. 122: 393-405.

Wood LC 1975 Thermodynamics of Fluid Systems. Oxford University Press, Oxford. 